\title{
Difusión de seis nuevas variedades de papa en Boyacá y Cundinamarca (Colombia) entre 2003 y 2010
}

\author{
Diffusion of six new potato varieties in Boyaca and \\ Cundinamarca (Colombia) between 2003 and 2010
}

JUAN CARLOS BARRIENTOS 1,3

CARLOS EDUARDO ÑÚSTEZ²

\section{Panorámica de cultivo de la variedad Pastusa Suprema en el municipio de La Calera (Cundinamarca). Foto: C.E. Nústez}

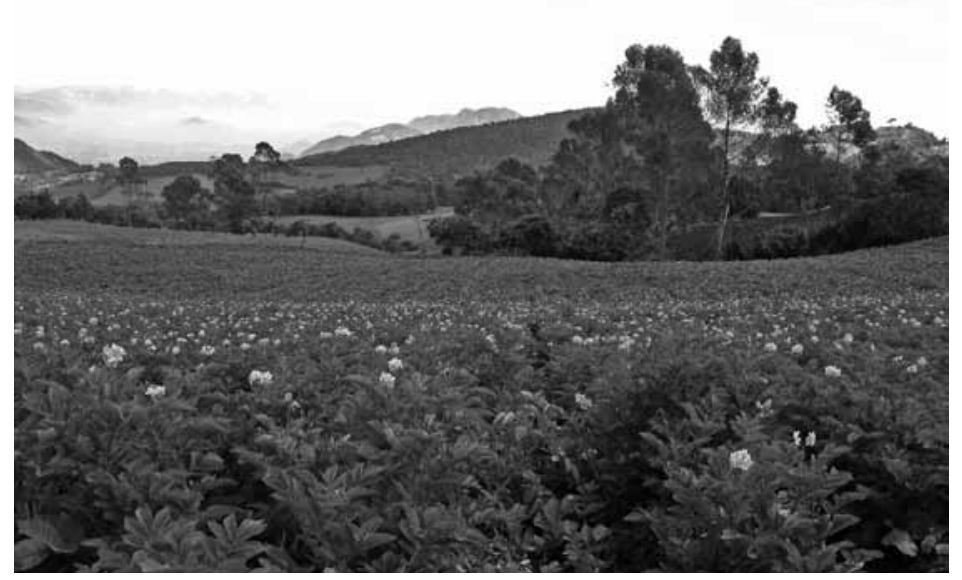

\section{RESUMEN}

Entre los años 2003 y 2005, la Universidad Nacional de Colombia, sede Bogotá, ha liberado al mercado de Boyacá y Cundinamarca seis nuevas variedades de papa tetraploide: Pastusa Suprema, Esmeralda, Betina, Punto Azul, Rubí y Roja Nariño. A pesar de que actualmente todas ya se encuentran en el mercado en diferente proporción, solo Pastusa Suprema aparece en las estadísticas oficiales de variedades cultivadas en los mencionados departamentos. En este sentido, el propósito de esta investigación es analizar la difusión de estas seis nuevas variedades de papa en Boyacá y Cundinamarca en el periodo 2003 a 2010. Para el logro de este cometido se hizo una encuesta a 800 productores en ambos departamentos y una entrevista a 15 expertos de la cadena de papa. Los resultados obtenidos son los siguientes: se identificaron seis canales de difusión, de los cuales sobresale el de los productores; la difusión obedeció principalmente a las directrices del mercado; las principales limitantes de la difusión fueron los precios de venta relativamente bajos, la escasa información sobre las nuevas variedades disponibles para consumidores y productores, y la escasa disponibilidad de semilla; la variedad más difundida y adoptada es Pastusa Suprema, seguida de Esmeralda y Betina, y luego por Punto Azul, Rubí y Roja Nariño; la superficie total de cultivo de las nuevas variedades se ha incrementado de forma sostenida hasta alcanzar en 2010 un 43\% de la superficie total de papa en Boyacá y 53\% en Cundinamarca. Adicionalmente, se ha determinado que las principales razones de adopción de las nuevas variedades fueron sus altos rendimientos y mayor resistencia a "gota" (Phytophthora infestans) comparado con las "variedades antiguas"; mientras que la escasa información y poca disponibilidad de semilla fueron razones relevantes para su no adopción.

1 Facultad de Ciencias Agrarias, Departamento de Desarrollo Rural, Universidad Nacional de Colombia, Bogotá (Colombia).

2 Facultad de Ciencias Agrarias, Departamento de Agronomía, Universidad Nacional de Colombia, Bogotá (Colombia).

3 Autor para correspondencia. jcbarrientosf@unal.edu.co 
Palabras clave adicionales: canales de difusión, adopción, superficie de cultivo, rendimientos, variedades tetraploides.

\section{ABSTRACT}

Between 2003 and 2005, the National University of Colombia, Bogotá released six new tetraploid potato varieties in the markets of the Boyacá and Cundinamarca Regions: Pastusa Suprema, Esmeralda, Betina, Punto Azul, Ruby and Roja Nariño. However, although all of them are currently present in the market in different proportions, only Pastusa Suprema appears in the official statistics of cultivated varieties in these Regions. In this sense, the purpose of this research was to analyze the diffusion of these six new varieties of potato in Boyacá and Cundinamarca (Colombia) between 2003 and 2010. For this purpose, a survey was carried out with 800 producers in both departments and 15 experts of the potato value chain were interviewed. The obtained results were as follows: six diffusion-channels were identified; the producers-channel was the most important. The diffusion was market-driven. The lack of information for consumers and producers, the low prices for these varieties, and the limited availability of seed were the main obstacles for diffusion. Pastusa Suprema is the most widespread and adopted variety, followed by Esmeralda and Betina, and finally Punto Azul, Rubí and Roja Nariño. The entire cultivated area of the new varieties has steadily increased, reaching $43 \%$ of the total potato area in Boyacá and 53\% in Cundinamarca for 2010. The main reasons for adoption iclude their high yields and greater resistance to late blight in comparison to "old varieties", while reasons for non-adoption are related to lack of information and unavailability of seed.

Additional key words: diffusion channels, adoption, crop area, yields, tetraploid varieties.

Fecha de recepción: 28-09-2013

Aprobado para publicación: 03-06-2014

(

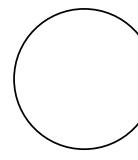

INTRODUCCIÓN

La papa es un producto de gran relevancia en la seguridad alimentaria, reducción de la pobreza, intensificación de los sistemas agrícolas sostenibles y aprovechamiento sostenible de la biodiversidad, razones por las que la Organización de Naciones Unidas declaró el 2008 como el Año Internacional de la Papa (FAO, 2009). Después del maíz, trigo y arroz, la papa es el alimento no cereal más consumido a nivel mundial. Se cultiva de norte a sur, y de este a oeste del planeta en 157 países, cuya producción total para 2010 fue superior a las 334 millones de toneladas. Asia y Europa son las regiones con mayor producción, y Europa y Norteamérica las de mayor consumo per cápita (FAO, 2009).
En Latinoamérica y el Caribe se cultiva papa en 25 países, de los cuales los de mayor producción para el año 2010 en orden de importancia fueron: Perú con 3.814.370 t, Brasil con 3.547.510 t, Colombia con 2.121.880 t, Argentina con 1.996 .040 t, y México con 1.536 .620 t (FAO, 2013). En Colombia, el cultivo de papa se encuentra entre los 10 más importantes del subsector de producción vegetal, y entre los 4 del grupo de los cultivos transitorios (Encuesta Nacional Agropecuaria 2009). Según Villarreal (comunicación personal, 2011), en la actualidad hay aproximadamente 90.000 familias dedicadas al cultivo de la papa, que en 2010 cubrió 136.664 ha a nivel nacional (Nústez, 2011). Los departamentos con mayor producción en orden 
Tabla 1. Producción (año 2010) y consumo (año 2005) de papa por regiones.

\begin{tabular}{|c|c|c|c|c|c|}
\hline \multirow[b]{2}{*}{ Región } & \multicolumn{3}{|c|}{ Producción 2010} & \multicolumn{2}{|c|}{ Consumo 2005} \\
\hline & $\begin{array}{l}\text { Área cosechada } \\
\text { (ha) }\end{array}$ & $\begin{array}{l}\text { Producción } \\
\text { (t) }\end{array}$ & $\begin{array}{l}\text { Rendimiento } \\
\left(\mathrm{t} \mathrm{ha}^{-1}\right)\end{array}$ & $(t)$ & $\mathrm{kg} /$ persona \\
\hline África & 1.827 .282 & 25.378 .948 & 13,89 & 12.571 .000 & 13,9 \\
\hline América del Norte & 547.868 & 22.760 .265 & 41,54 & 19.824 .000 & 60,0 \\
\hline América Central & 81.463 & 2.151 .715 & 26,41 & \multirow{3}{*}{11.639 .000} & \multirow{3}{*}{20,7} \\
\hline Caribe & 12.921 & 262.205 & 20,29 & & \\
\hline América del Sur & 947.203 & 14.550 .343 & 15,36 & & \\
\hline Asia & 9.193 .486 & 159.055 .291 & 17,30 & \multirow{2}{*}{94.038 .800} & \multirow{2}{*}{23,9} \\
\hline Oceanía & 49.458 & 1.871 .481 & 37,84 & & \\
\hline Europa & 6.109 .489 & 108.232.274 & 17,72 & 64.902 .000 & 87,8 \\
\hline Mundo & 18.769.170 & 334.262 .523 & 17,81 & 202.974 .000 & 31,3 \\
\hline
\end{tabular}

Fuente: FAOSTAT, 2013; FAO, 2009.

de importancia son Cundinamarca, Boyacá, Nariño y Antioquia, de los cuales los dos primeros aportan cerca del 70\% de la producción nacional. Las variedades que se han destacado en los últimos años por sus porcentajes de participación en la producción total nacional por orden de importancia son Pastusa Suprema, Diacol Capiro y Parda Pastusa. La primera es una variedad relativamente nueva que fue reemplazando paulatinamente a la última.

Entre los años 2001 y 2003 se realizó el primer censo nacional de la papa, cuya información resultante se constituyó en una línea base para analizar y abordar los problemas de este cultivo (Ministerio de Agricultura y Desarrollo Rural, DNP y DANE, 2003a y 2003b). Los resultados más relevantes del mencionado documento se presentan en la tabla 2 .

El programa de mejoramiento genético de papa de la Universidad Nacional de Colombia tuvo el propósito de mejorar la competitividad de la cadena de papa y, en consecuencia, las condiciones socioeconómicas de los productores. Desde 1998 se trabaja en el desarrollo de nuevas variedades de papa, entre otros, para incrementar el rendimiento del cultivo, disminuir las pérdidas por enfermedades e incursionar en nuevos mercados. En la fase final con clones avanzados realizó un proceso de investigación participativa con productores, posteriormente registró seis nuevas variedades de papa tetraploide para Colombia entre los años 2002 y 2005. Las variedades Betina, Pastusa Suprema y Roja Nariño fueron entregadas a los agricultores para su aprovechamiento comercial en diciembre de 2002, en tanto que Esmeralda, Punto Azul y Rubí en noviembre de 2005. Las variedades Pastusa Suprema, Betina y Roja Nariño fueron registradas ante el ICA para la Subregión Nudo de los Pastos.

Las estadísticas oficiales de producción, tanto del gremio papero como del Estado, no muestran la presencia de estas nuevas variedades en los datos de los últimos años, excepto el de 'Pastusa Suprema'. Si bien la entrega de las variedades en cuestión a los agricultores no fue acompañada de un programa formal de difusión (extensión), es posible encontrar en el mercado la mayoría de ellas, lo cual indica que a pesar de todo se ha llevado a cabo un proceso de difusión; lo que transforma la composición varietal 
Tabla 2. Principales resultados del primer censo nacional de la papa en Boyacá y Cundinamarca.

\begin{tabular}{|c|c|c|}
\hline \multirow[b]{2}{*}{ Características del sistema de producción } & \multicolumn{2}{|c|}{ DEPARTAMENTOS } \\
\hline & $\begin{array}{c}\text { Boyacá } \\
\text { (junio 2002 - junio 2003) }\end{array}$ & $\begin{array}{l}\text { Cundinamarca } \\
\text { (julio } 2001 \text { - junio 2002) } \\
\end{array}$ \\
\hline Superficie promedio (ha) & 0,64 & 2,23 \\
\hline Productores con cultivo de papa menor a 1 ha & $68 \%$ & $65 \%$ \\
\hline $\begin{array}{l}\text { Número total de productores } \\
\text { de papa por departamento }\end{array}$ & 47.592 & 25.256 \\
\hline Variedades y rendimientos & $\begin{array}{l}\text { Parda Pastusa y otras } 17,9 \mathrm{t} \mathrm{ha}^{-1} \\
\text { Industriales } 22,4 \mathrm{t} \mathrm{ha}^{-1} \\
\text { Criolla } 8,4 \mathrm{t} \mathrm{ha}^{-1}\end{array}$ & $\begin{array}{l}\text { Parda Pastusa y otras } 18,7 \mathrm{t} \mathrm{ha}^{-1} \\
\text { Industriales } 23,1 \mathrm{t} \mathrm{ha}^{-1} \\
\text { Criolla } 15,3 \mathrm{t} \mathrm{ha}^{-1}\end{array}$ \\
\hline $\begin{array}{l}\text { Participación porcentual de variedades } \\
\text { respecto de la superficie total de cultivo }\end{array}$ & $\begin{array}{l}\text { Parda Pastusa y otras 68\% } \\
\text { Diacol Capiro } 30 \% \\
\text { Criolla } 2 \%\end{array}$ & $\begin{array}{l}\text { Parda Pastusa y otras } 74 \% \\
\text { Diacol Capiro } 18 \% \\
\text { Criolla } 8 \%\end{array}$ \\
\hline Rango de alturas del cultivo de papa & Sin información & $\begin{array}{l}93 \% \text { entre } 2.500 \text { y } 3.500 \mathrm{msnm} \\
40 \%>3.000 \mathrm{msnm}\end{array}$ \\
\hline Destino de la producción & $\begin{array}{l}84 \% \text { para mercado } \\
8 \% \text { para autoconsumo } \\
8 \% \text { para semilla }\end{array}$ & $\begin{array}{l}89 \% \text { para mercado } \\
7 \% \text { para autoconsumo } \\
4 \% \text { para semilla }\end{array}$ \\
\hline Otras características del sistema & $93 \%$ sin riego & $\begin{array}{l}89 \% \text { sin asistencia técnica } \\
65 \% \text { sin crédito bancario } \\
81 \% \text { sin riego }\end{array}$ \\
\hline
\end{tabular}

Fuente: Ministerio de Agricultura y Desarrollo Rural, DNP y DANE, 2003a y 2003b.

del producto papa en el mercado colombiano. De este hecho surgen las siguientes preguntas que han dado pie a la presente investigación: ihan sido adoptadas todas las variedades en cuestión?, żcómo y en qué medida ha transcurrido su adopción?, żqué dificultades se han presentado? y i̇cuáles son sus proyecciones?

El objetivo principal de la investigación fue examinar la difusión de las seis variedades de papa liberadas por el programa de mejoramiento genético de la UNC en los departamentos de Boyacá y Cundinamarca (Colombia) entre 2003 y 2010. Como objetivos secundarios se plantearon: (a) describir el proceso de difusión de las nuevas variedades, (b) determinar el grado y características de adopción, (c) identificar las razones para la adopción y no adopción de las nuevas variedades, (d) determinar la superficie de cultivo alcanzada por las nuevas variedades y (e) hacer una proyección de la adopción de las nuevas variedades después de 2010.

\section{METODOLOGÍA}

\section{Requerimiento de información y fuentes}

\section{Información cualitativa}

Las fuentes de información para la descripción del proceso de difusión que siguieron las nuevas variedades fueron los llamados "expertos" y material bibliográfico con información secundaria. 


\section{Información cuantitativa}

Se estimó el porcentaje y grado de adopción, la superficie de cultivo para el año 2010 y las razones de adopción y no adopción. Las fuentes de información fueron los productores de papa. La información se obtuvo mediante encuesta a productores.

\section{Obtención de la información}

\section{Muestreo}

Se tomó como base de información el I Censo Nacional de la Papa. El muestreo para cada departamento fue estratificado. Primero se eligieron municipios y luego productores (fincas). Por cuestiones logísticas se excluyeron del universo a los municipios (y sus productores) con participación porcentual en superficie de cultivo menor o igual a $1 \%$ respecto del total del departamento. Así, de una población de 61 municipios paperos de Cundinamarca se seleccionaron solo 23 , que cubren el $86 \%$ del área total de cultivo; y de una población de 81 municipios de Boyacá se seleccionaron solo 27, que cubren casi el $80 \%$ de la superficie total de cultivo. Los productores de los municipios seleccionados forman la población a muestrear. Para determinar el número de encuestas por departamento se utilizó la fórmula:

$n=\frac{N \cdot z^{2} \cdot p \cdot q}{\left(N \cdot e^{2}\right)+\left(z^{2} \cdot p \cdot q\right)}$

donde:

$\mathrm{n}=$ tamaño de la muestra.

$\mathrm{N}=$ tamaño de la población (31.133 productores en Boyacá y 19.041 productores en Cundinamarca.

$\mathrm{e}=$ error de la muestra $(5 \%=0,05)$. $z=$ nivel de confianza $(95 \%=1,96)$

$\mathrm{p}=$ proporción de productores con una nueva variedad $=0,5$.

$\mathrm{q}=$ proporción de productores sin una nueva variedad $=0,5$.

$n_{\text {Boyacá }}=\frac{31.133 \times(1,96)^{2} \times 0,5 \times 0,5}{\left(31.133 \times(0,05)^{2}\right)+\left((1,96)^{2} \times 0,5 \times 0,5\right)}=379,5$

$n_{\text {Cundinamarca }}=\frac{19.041 \times(1,96)^{2} \times 0,5 \times 0,5}{\left(19.041 \times(0,05)^{2}\right)+\left((1,96)^{2} \times 0,5 \times 0,5\right)}=376,6$

Al final, el número de unidades muestreales en campo se redondearon a 800 productores, en Cundinamarca 398 y en Boyacá 402, ambos con un error muestral de 4,86\%. El número de productores a encuestar en cada municipio (estrato) se calculó con base en la proporción porcentual de productores que había en cada municipio respecto de la población de productores del departamento. Por ejemplo, el municipio Samacá de Boyacá tiene, según el primer censo papero de 2002, 1.162 fincas (productores), el 4\% de la población papera (31.133 fincas), lo que dio 16 productores para encuestar.

\section{Encuesta}

Entre mediados de enero y finales de febrero de 2011 se encuestaron 800 productores, por medio de un cuestionario estructurado con alto porcentaje de preguntas cerradas, basado en un modelo de encuesta propuesto por el Centro Internacional de la Papa (CIP, Perú) en el caso de la variedad Serranita INIA-309, al cual se le realizaron los ajustes respectivos teniendo en cuenta el sistema productivo en Colombia.

\section{Entrevista a expertos}

Entre finales de febrero y finales de abril de 2011 se hicieron entrevistas personales cara a 
cara utilizando cuestionarios semiestructurados con preguntas abiertas a 15 agentes de la cadena de papa: secretario técnico del Consejo Nacional de la Papa, un técnico de campo de Fedepapa, un comercializador de agroinsumos, dos miembros de cooperativas paperas de Boyacá, dos productores grandes de papa, un empresario productor de semilla, un industrial de la papa, cinco comercializadores y un investigador. Estas personas fueron elegidas por su experticia en el área o eslabón de cadena que representan.

\section{Análisis de la información}

La información recolectada en las encuestas se sistematizó en una hoja Excel y se preparó una base de datos. El análisis que se realizó fue en su mayoría de tipo descriptivo. La información de los expertos se clasificó y organizó en categorías, que se utilizaron para complementar y detallar la información resultante de la encuesta.

\section{RESULTADOS Y DISCUSIÓN}

\section{Canales y métodos de difusión de las nuevas variedades}

La difusión de innovaciones tecnológicas en el ramo papero se realiza de manera formal principalmente a través de las llamadas Escuelas de Campo de Agricultores (ECA) a cargo de la Federación Colombiana de Productores de Papa (Fedepapa). Sin embargo, esta forma de difusión, por los pocos recursos disponibles en el gremio, ha llegado apenas a un $5 \%$ de los productores en 10 años (Villarreal, comunicación personal, 2011). En este contexto, el grupo de investigación en papa de la UNC, sin recursos para financiar un proceso de difusión controlado, en el caso de Cundinamarca y Boyacá, ha iniciado la difusión de las seis variedades en estudio recurriendo a canales semiprivados y privados, que son: (1) grandes y medianos productores de papa, (2) almacenes de expendio de insumos agropecuarios (Fedepapa), (3) organizaciones de productores

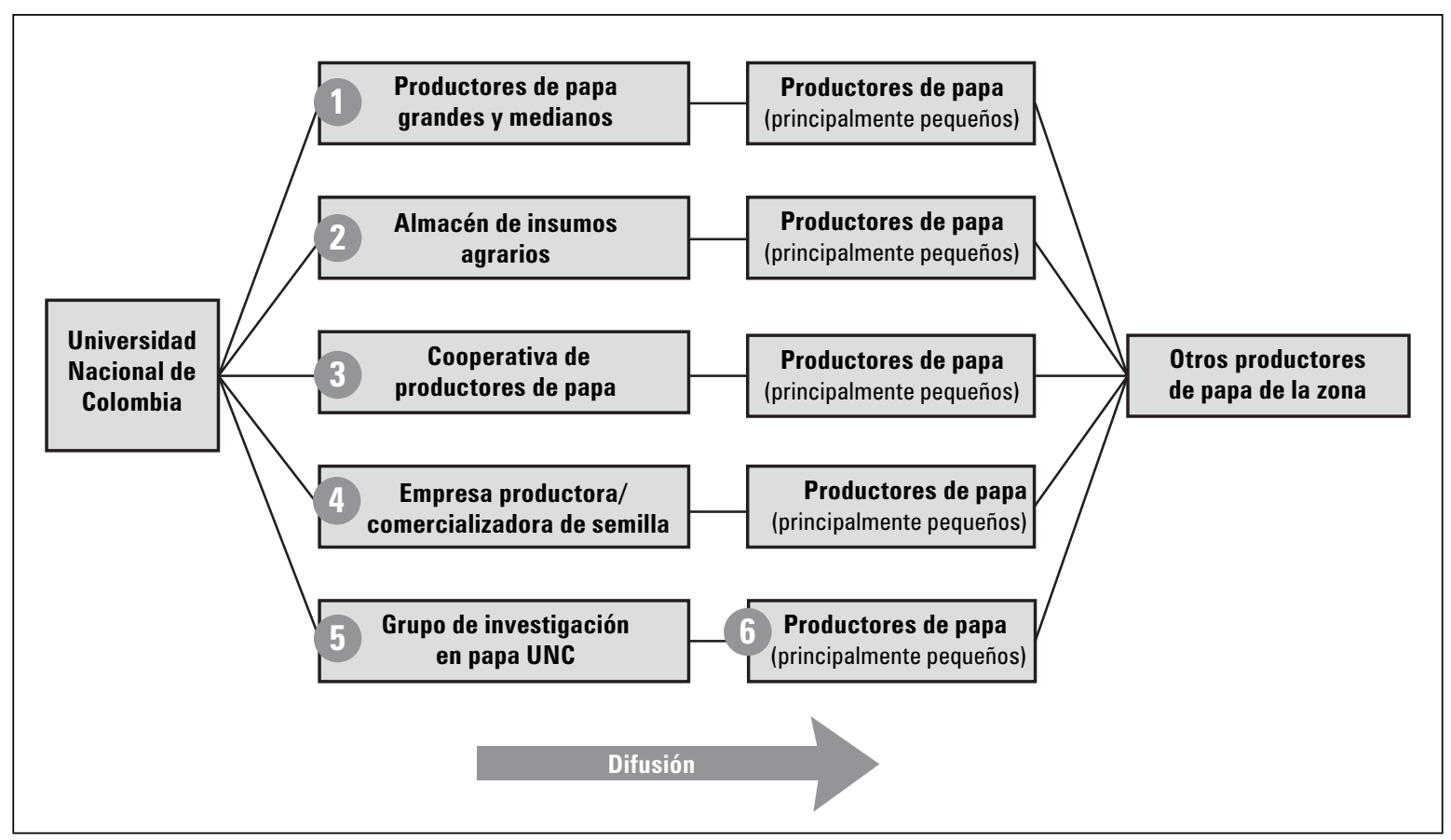

Figura 1. Canales de difusión utilizados en Boyacá y Cundinamarca para las seis nuevas variedades de papa entre 2003 y 2010. 
de papa, (4) empresa productora de semillas, (5) grupo de investigación en papa y (6) pequeños productores (figura 1). En todos los casos, el impulso o freno de la difusión y adopción ha sido principalmente el mercado a través del precio, la oferta de otras variedades y la demanda del intermediario y del consumidor.

Según Rogers (2003), la difusión de innovaciones tiene cuatro elementos básicos: la innovación como tal, el canal de comunicación, el tiempo y el sistema social donde ocurre este proceso. El canal de comunicación transmite información acerca de la innovación; sin embargo, la difusión implica también la transferencia del producto como tal (bien o servicio). El camino que toma la transferencia de información y producto se le denomina "canal de difusión", que puede estar constituido por entes estatales, semiprivados o privados. La difusión de innovaciones agrarias en Latinoamérica y otros países en desarrollo fue llevada a cabo principalmente por el Estado a través de centros de investigación y proyectos o instituciones de extensión, especialmente entre las décadas de 1950 y 1990 (Acasio et al., 1986; Bejarano et al., 2004; Esprella et al., 2002; Mauceri et al., 2005). Sin embargo, en las últimas décadas esta actividad fue pasando a manos semiprivadas y privadas como ONG, gremios y empresas agrarias (Morris et al., 2003). Esta tendencia se ha manifestado también en el sector agrario colombiano, y específicamente en el ramo papero.

\section{Difusión a través de productores}

Los productores grandes y medianos, al poseer mayor capital que los pequeños, manejan mayores volúmenes de producto, lo que les confiere un mayor grado de poder de negociación en el mercado, tanto de semilla como de papa para consumo. Si ellos identifican ventajas competitivas adoptan más rápido y pueden influir de manera más directa en los intermediarios y consumidores. Todos los productores se convierten en fuente de provisión de semilla no formal, la cual se distribuye principalmente entre sus vecinos. Esta ha sido la forma más frecuente de difusión de las nuevas variedades.

\section{Difusión a través de almacén de insumos, organi- zaciones de productores y empresa productora de semilla}

Estos tres agentes responden a la oferta del proveedor y a la demanda del comprador (productor o intermediario) de semilla de variedades nuevas. Ambas estrategias, denominadas push and pull en mercadeo, han estado poco presentes en estos canales, lo que determinó una reducida participación en la difusión de las nuevas variedades. Otro factor que pudo influir en la reducida participación de estos canales fue la ausencia del registro ICA de las variedades para la región cundiboyacense hasta el año de estudio.

\section{Difusión a través del grupo de investigación en papa de la UNC}

El grupo de investigación en papa tuvo una participación activa al principio, durante la entrega a productores de la semilla de las nuevas variedades. Posteriormente, su rol en la difusión ha sido poco más que pasiva, esto debido a dos factores: (1) no es su función y (2) no cuenta con recursos suficientes para esta actividad. Aun así, este grupo provee semilla a los productores que la demandan, principalmente semillas prebásicas como superélite y élite. Dado que la red de comunicación entre los productores y el grupo de investigación es reducida, la demanda de semilla y la difusión han sido también escasas.

\section{Métodos de difusión}

Al inicio del proceso se utilizaron parcelas demostrativas y días de campo para dar a conocer a los productores las nuevas variedades y sus características. Se utilizaron hojas divulgativas de las variedades las cuales se distribuyeron en reuniones con diferentes actores, y también se ha utilizado la página web del grupo (www.papaunc.com). 
Tabla 3. Estrategias de difusión de las nuevas variedades de papa.

\begin{tabular}{|c|c|c|c|c|}
\hline Agentes & Zona de influencia & $\begin{array}{l}\text { Variedad de } \\
\text { papa }\end{array}$ & Año & Estrategias/métodos \\
\hline Productor grande & $\begin{array}{l}\text { Municipio Zipaquirá } \\
\text { (Cundinamarca) }\end{array}$ & $\begin{array}{l}\text { Pastusa } \\
\text { Suprema }\end{array}$ & 2003 & $\begin{array}{l}\text { - Cultivo extensivo. } \\
\text { - Día de campo para vender } \\
\text { semilla a productores de la zona. } \\
\text { - Comercialización de grandes } \\
\text { volúmenes en mercados mayoristas. }\end{array}$ \\
\hline Productor mediano & $\begin{array}{l}\text { Municipio Villapinzón } \\
\text { (Cundinamarca) }\end{array}$ & Roja Nariño & 2008 & $\begin{array}{l}\text { - Cultivo extensivo y eficiente } \\
\text { - Comercialización de grandes } \\
\text { volúmenes en mercados mayoristas. }\end{array}$ \\
\hline Productor pequeño & $\begin{array}{l}\text { Boyacá y } \\
\text { Cundinamarca }\end{array}$ & Todas & $\begin{array}{l}2004 \text { y } \\
2005\end{array}$ & $\begin{array}{l}\text { - Producción y distribución de semilla a } \\
\text { vecinos productores de papa de la zona. }\end{array}$ \\
\hline $\begin{array}{l}\text { Almacén de } \\
\text { insumos agrarios }\end{array}$ & $\begin{array}{l}\text { Municipio Villapinzón } \\
\text { (Cundinamarca) }\end{array}$ & Betina & 2003 & $\begin{array}{l}\text { - Venta de semilla a productores } \\
\text { de Boyacá y Cundinamarca. }\end{array}$ \\
\hline $\begin{array}{l}\text { Cooperativa } \\
\text { COINPAVEN }\end{array}$ & $\begin{array}{l}\text { Municipio } \\
\text { Ventaquemada } \\
\text { (Boyacá) }\end{array}$ & $\begin{array}{l}\text { Punto Azul y } \\
\text { Betina }\end{array}$ & 2005 & $\begin{array}{l}\text { - Producción y distribución } \\
\text { de semilla entre socios. } \\
\text { - Producción y venta de semilla. }\end{array}$ \\
\hline $\begin{array}{l}\text { Cooperativa } \\
\text { COPABOY }\end{array}$ & $\begin{array}{l}\text { Municipio Tunja } \\
\text { (Boyacá) }\end{array}$ & $\begin{array}{l}\text { Esmeralda, } \\
\text { Punto Azul y } \\
\text { Rubí }\end{array}$ & 2005 & $\begin{array}{l}\text { - Ensayos en parcelas demostrativas. } \\
\text { - Distribución de semilla entre socios. } \\
\text { - Día de campo. }\end{array}$ \\
\hline $\begin{array}{l}\text { Grupo de } \\
\text { investigación en } \\
\text { papa de la UNC }\end{array}$ & $\begin{array}{l}\text { Bogotá } \\
\text { (Cundinamarca) }\end{array}$ & Todas & $\begin{array}{l}2003 \text { y } \\
2005\end{array}$ & $\begin{array}{l}\text { - Multiplicación de semilla prebásica. } \\
\text { - Distribución (venta) de semilla entre } \\
\text { productores. }\end{array}$ \\
\hline $\begin{array}{l}\text { Empresa productora } \\
\text { y comercializadora } \\
\text { de semilla }\end{array}$ & $\begin{array}{l}\text { Boyacá y } \\
\text { Cundinamarca }\end{array}$ & $\begin{array}{l}\text { Pastusa } \\
\text { Suprema, } \\
\text { Esmeralda y } \\
\text { Rubí }\end{array}$ & 2010 & $\begin{array}{l}\text { - Producción y venta de semilla entre } \\
\text { productores de la zona. } \\
\text { - Días de campo. } \\
\text { - Capacitaciones a agricultores. }\end{array}$ \\
\hline
\end{tabular}

\section{Limitantes de la difusión}

El relativo bajo grado de adopción de las nuevas variedades por parte de los productores ha limitado en gran medida la difusión de los mencionados materiales. Las principales causas para que suceda esto fueron: (1) El poder de negociación ejercido por comerciantes intermediarios y mayoristas, quienes generalmente determinan los precios de las nuevas variedades a su favor en detrimento de los productores, sobre todo de los pequeños; esto disminuye la rentabilidad del cultivo, lo que limita la demanda de semilla, y en consecuencia de su producción. (2) La información técnica de las nuevas variedades como rendimiento, tolerancia a enfermedades y otras cualidades de interés no les llega a muchos productores. (3) La poca disponibilidad de semilla certificada no permite cubrir su demanda real y potencial. (4) Otros factores, entre ellos se pueden mencionar: la dispersión geográfica de los productores, el uso tradicional de variedades antiguas y la poca información (promoción) que tienen los consumidores finales acerca de las nuevas variedades. 


\section{Porcentaje y grado de adopción de las nuevas variedades}

Desde su liberación las seis nuevas variedades han tenido un grado y porcentaje de adopción diferente. En ambos departamentos se destaca Pastusa Suprema, seguida de Esmeralda, Betina, Punto Azul y Rubí. Aunque en Villapinzón se reportó la siembra de Roja Nariño, su porcentaje de adopción es tan bajo que todavía aparece como cero (figuras 2 y 3 ).

La principal razón del éxito en la adopción de Pastusa Suprema es el parecido de su tubérculo al de la variedad más tradicional y consumida en el centro del país, Parda Pastusa, pero también por su alto rendimiento y su respuesta de resistencia a la 'gota' (Phytophthora infestans), por el tiempo que lleva en mercado, así como también por el canal de difusión que tomó, que es el de gran productor. La variedad Esmeralda se difundió también de manera relativamente exitosa debido a que los productores y comercializadores utilizaron como base el nombre de un cultivar, 'Marengo', ya presente en el mercado, cuyo fenotipo es relativamente similar, denominándola 'Marengo-chiquita' o 'Marenguita', lo que al principio generó curiosidad entre los compradores, quienes luego se convencieron por su calidad culinaria. Las variedades Betina, Punto Azul, Rubí y Roja Nariño se difundieron a un ritmo más lento y en volúmenes menores debido principalmente a los siguientes aspectos: (1) tienen fenotipos de tubérculo relativamente

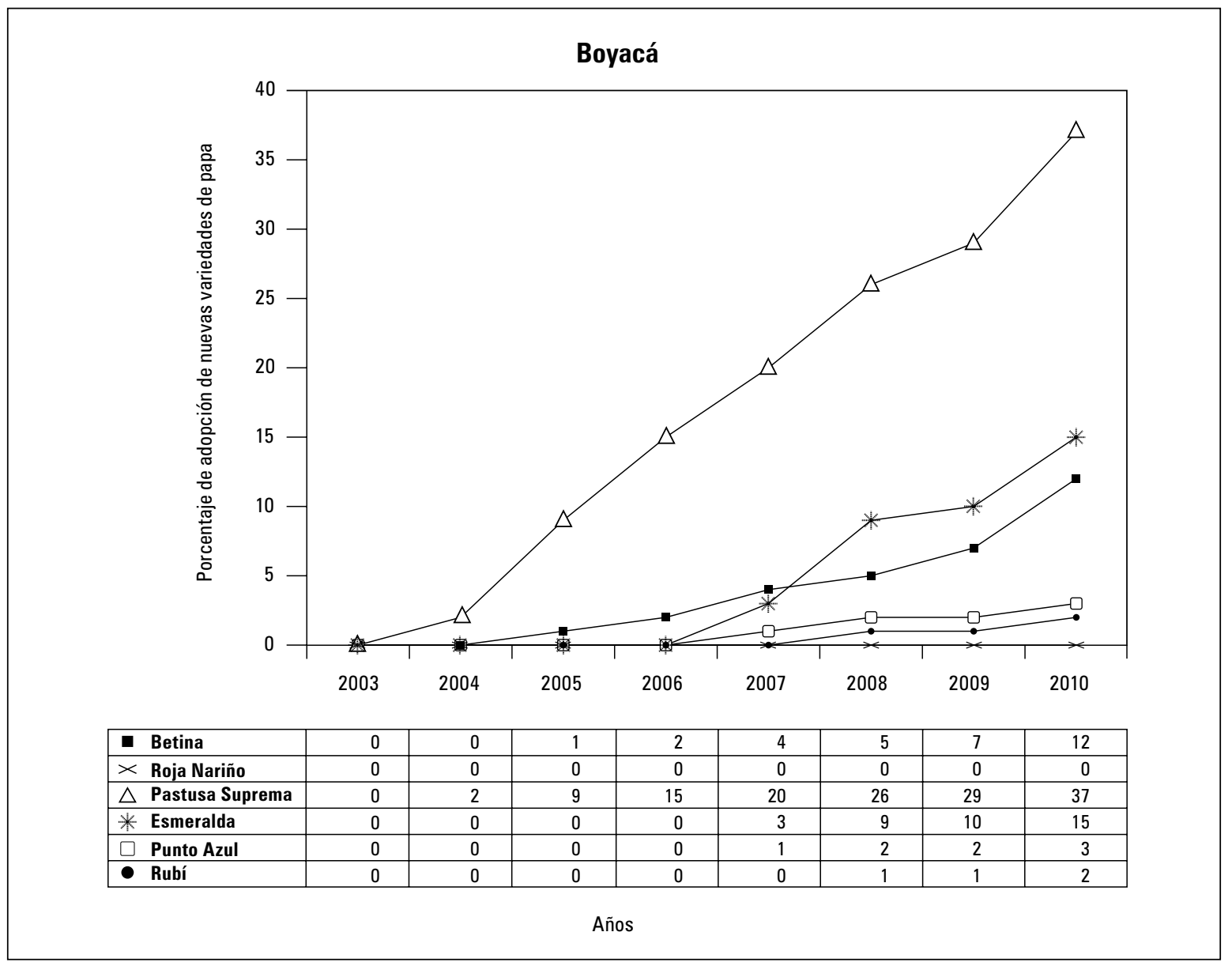

Figura 2. Porcentaje de adopción de nuevas variedades de papa en Boyacá entre 2003 y 2010. 


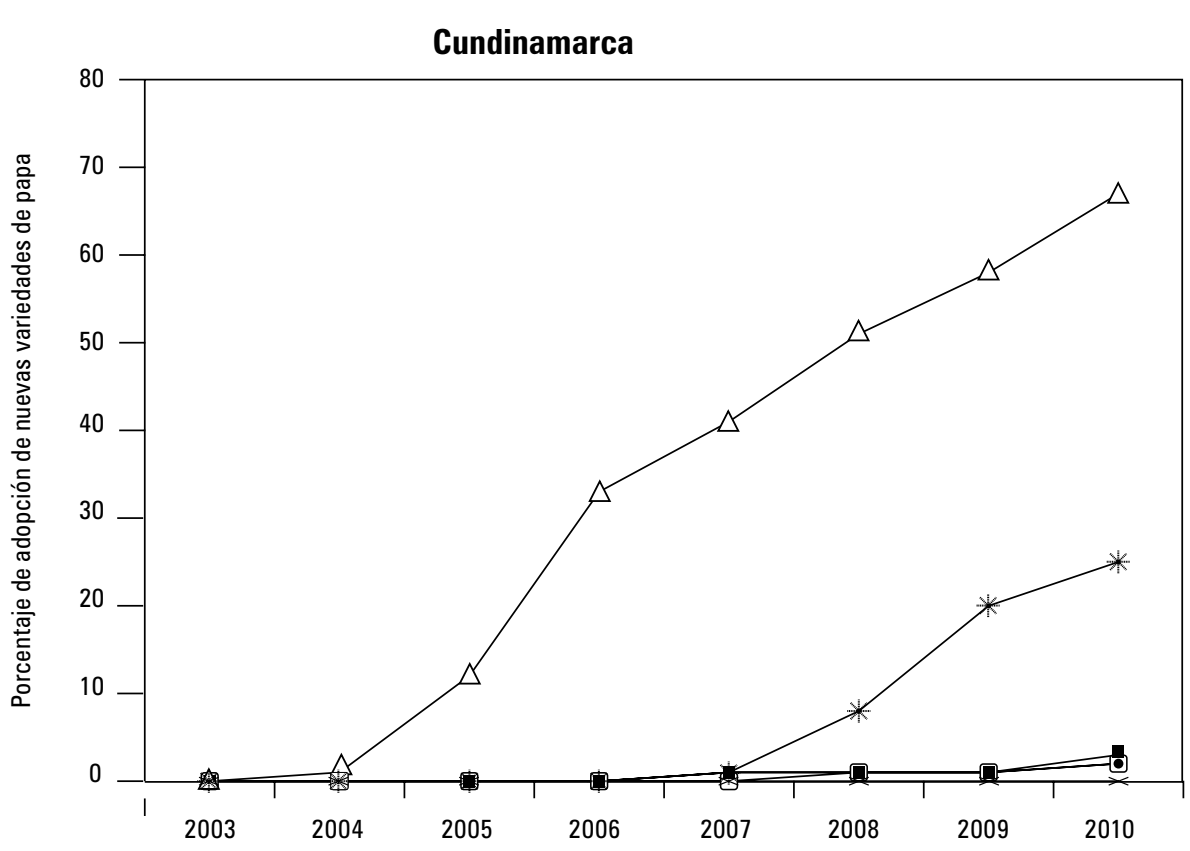

\begin{tabular}{|l|r|r|r|r|r|r|r|r|}
\hline — Betina & 0 & 0 & 0 & 0 & 1 & 1 & 1 & 3 \\
\hline$\times$ Roja Nariño & 0 & 0 & 0 & 0 & 0 & 0 & 0 & 0 \\
\hline$\triangle$ Pastusa Suprema & 0 & 1 & 12 & 33 & 41 & 51 & 58 & 67 \\
\hline$*$ Esmeralda & 0 & 0 & 0 & 0 & 1 & 8 & 20 & 25 \\
\hline$\square$ Punto Azul & 0 & 0 & 0 & 0 & 0 & 1 & 1 & 2 \\
\hline$\bullet$ Rubí & 0 & 0 & 0 & 0 & 1 & 1 & 1 & 2 \\
\hline
\end{tabular}

Años

Figura 3. Porcentaje de adopción de nuevas variedades de papa en Cundinamarca entre 2003 y 2010.

diferentes a las variedades tradicionales, (2) no han existido agricultores grandes que impulsen su producción y presencia en los mercados de manera significativa y continua, (3) no ha habido procesos estructurados de difusión por lo que muchos agricultores no las conocen, y (4) los consumidores, e inclusive productores, no han tenido muchas oportunidades para probarlas, en su gran mayoría no saben que existen (figura 7).

En Cundinamarca se observa una mayor adopción debido principalmente a dos factores: (1) las unidades productivas tienen un perfil más comercial que en Boyacá y (2) la dinámica comercial es mayor por estar más cerca del mercado colombiano más grande de alimentos, Bogotá.
La velocidad de adopción fue relativamente alta para Pastusa Suprema, en promedio cerca de 10\% por año. Las otras variedades han sido adoptadas a una velocidad media a lenta. El grado de adopción de las nuevas variedades es relativamente baja, es decir 2,4\% anual en promedio. Se adopta la semilla, pero no la tecnología que acompaña a ese producto: menor uso de plaguicidas, diversos rangos de altitud para su siembra y calidad culinaria; esto debido a las deficiencias en la transferencia de tecnología, que está asociada a la falta de recursos para esta fase.

Cuando se introduce una innovación en un sistema social, no todos la adoptan al mismo tiempo y grado. Según Rogers (2003), de acuerdo con tiempo que necesitan para adoptar una 
innovación, hay cinco tipos de adoptadores: (1) innovadores (2,5\%), (2) adoptadores tempranos (13,5\%), (3) mayoría temprana (34\%), (4) mayoría tardía (34\%) y (5) rezagados (16\%). La adopción de innovaciones agrarias es mayormente parcial y de mediano/largo plazo. En un listado de 28 diferentes agroproductos se ha encontrado un rango de 4 a 41 años de tiempo y de 10 a 100\% de adopción (Barrientos y Berg, 2013). El resultado encontrado en esta investigación evidencia que las diferentes variedades se encuentran en diferentes fases y grados de adopción como lo muestran las figuras 2 y 3 . Cada variedad tiene un ritmo propio de adopción.

\section{Superficie de producción de las nuevas variedades}

Durante la última década la oferta nacional de papa se ha mantenido relativamente estable con alrededor de 2.700.000 t año-1; sin embargo al incrementarse el rendimiento promedio (de 18,2 en 2002 a 19,1 t ha $a^{-1}$ en 2010) ha disminuido su superficie de cultivo (tabla 4), así como

Tabla 4. Área de producción de papa (ha) en Boyacá, Cundinamarca y Colombia (2002-2010).

\begin{tabular}{|c|c|c|c|c|c|c|c|c|c|}
\hline Departamento & 12002 & 2003 & 2004 & 2005 & 2006 & 2007 & 2008 & ${ }^{2} 2009$ & ${ }^{12010}$ \\
\hline Boyacá & 41.603 & 341.666 & 341.103 & 338.559 & 340.143 & 340.803 & 335.121 & 36.820 & 37.370 \\
\hline Cundinamarca & 63.982 & ${ }^{3} 64.078$ & 363.213 & 359.301 & 361.737 & ${ }^{3} 62.751$ & 354.014 & 52.825 & 53.629 \\
\hline Otros departamentos & 58.256 & 58.344 & 57.557 & 53.994 & 56.211 & 57.136 & 49.180 & 44.995 & 45.665 \\
\hline Colombia $^{1}$ & 163.841 & 164.088 & 161.873 & 151.854 & 158.091 & 160.690 & 138.315 & 134.640 & 136.664 \\
\hline
\end{tabular}

Tabla 5. Área de cultivo de papa por variedades y años (2002 y 2009) en Boyacá y Cundinamarca.

\begin{tabular}{|c|c|c|c|c|c|c|c|c|c|}
\hline \multicolumn{5}{|c|}{ Área de cultivo de papa en $2002^{1}$} & \multicolumn{5}{|c|}{ Área de cultivo de papa en $2009^{2}$} \\
\hline \multirow{3}{*}{ Variedades } & \multicolumn{4}{|c|}{ Departamentos } & \multirow{3}{*}{ Variedades } & \multicolumn{4}{|c|}{ Departamentos } \\
\hline & \multicolumn{2}{|c|}{ Boyacá } & \multicolumn{2}{|c|}{ Cundinamarca } & & \multicolumn{2}{|c|}{ Boyacá } & \multicolumn{2}{|c|}{ Cundinamarca } \\
\hline & (ha) & $(\%)$ & (ha) & $(\%)$ & & (ha) & $(\%)$ & (ha) & $(\%)$ \\
\hline Criolla & 1.639 & 4 & 3.383 & 5 & Criolla & 1.841 & 5 & 2.641 & 5 \\
\hline Diacol Capiro (R12) & 8.870 & 21 & 11.529 & 18 & Diacol Capiro (R12) & 7.732 & 21 & 9.509 & 18 \\
\hline Diacol Monserrate & 81 & 0 & 81 & 0 & ICA Única & 1.841 & 5 & 1.056 & 2 \\
\hline ICA Chitaga & 36 & 0 & 61 & 0 & Parda Pastusa & 7.364 & 20 & 11.622 & 22 \\
\hline ICA Morita & 214 & 1 & 131 & 0 & Pastusa Suprema & 12.887 & 35 & 26.941 & 51 \\
\hline ICA Nariño & 178 & 0 & 115 & 0 & $\begin{array}{l}\text { Tuquerreña } 0 \\
\text { Sabanera }\end{array}$ & 4.418 & 12 & 528 & 1 \\
\hline ICA Puracé & 975 & 2 & 289 & 1 & Otras & 737 & 2 & 528 & 1 \\
\hline Parda Pastusa & 20.707 & 50 & 47.133 & 74 & TOTAL & 36.820 & 100 & 52.825 & 100 \\
\hline $\begin{array}{l}\text { Tuquerreña } 0 \\
\text { Sabanera }\end{array}$ & 5.034 & 12 & 198 & 0 & & & & & \\
\hline Única & 1.927 & 5 & 674 & 1 & & & & & \\
\hline Otras & 1.943 & 5 & 389 & 1 & & & & & \\
\hline TOTAL & 41.603 & 100 & 63.982 & 100 & & & & & \\
\hline
\end{tabular}

Fuente: 'I Censo Nacional de la Papa (2003); ${ }^{2}$ CNP (2010). 


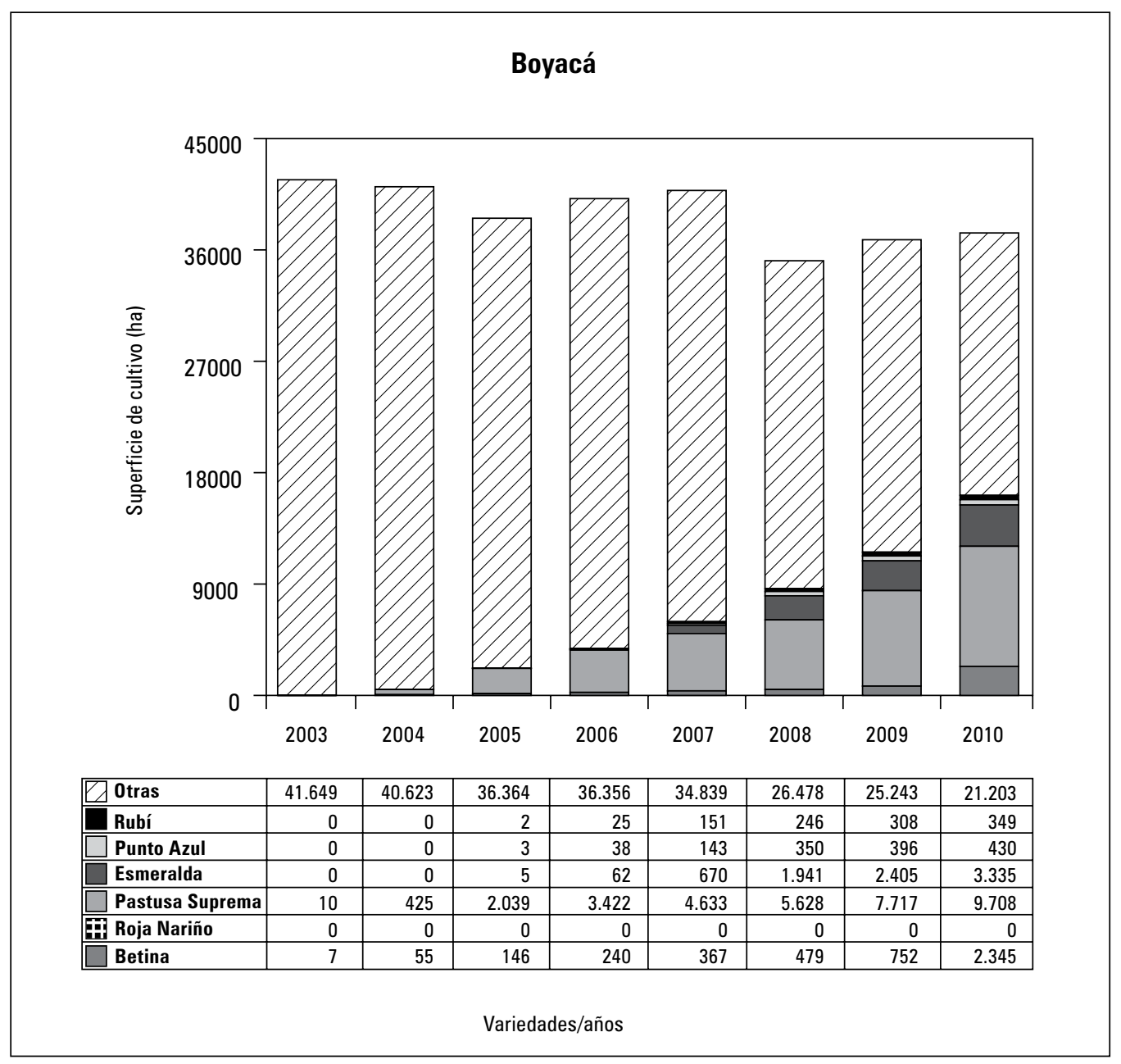

Figura 4. Área de cultivo de nuevas y antiguas (otras) variedades de papa en el departamento Boyacá entre 2003 y 2010.

su consumo per cápita de 76,4 en 1995 a 58 kg/ persona/año en 2008 (CNP, 2010).

El Observatorio Agrocadenas Colombia (2005) del Ministerio de Agricultura y Desarrollo Rural ha reportado que a nivel nacional habría 30 variedades de papa, de las cuales 10 tendrían mayor importancia comercial. La estructura varietal de papa ha ido cambiando con los años y con la aparición y difusión de nuevas variedades (tabla 5, figuras 4 y 5 ).

Desde el año de su entrega a agricultores, la superficie de cultivo de las nuevas variedades se incrementó de forma sostenida hasta alcanzar en 2010 un 43\% de la superficie total en Boyacá y $53 \%$ en Cundinamarca (figuras 4 y 5 ).

Paralelo al proceso de adopción, las variedades que se han incrementado más en superficie de cultivo son, en orden descendente: Pastusa Suprema, Esmeralda y Betina. Las otras tres variedades aún ocupan superficies menores a mil hectáreas, pero con tendencia a incrementar su área. Este es un resultado muy relevante para el programa de mejoramiento genético de papa de la UNC, que en un periodo relativamente corto ha evidenciado su impacto en la transformación del sistema productivo de papa en la región de estudio, principal zona productora del país. 


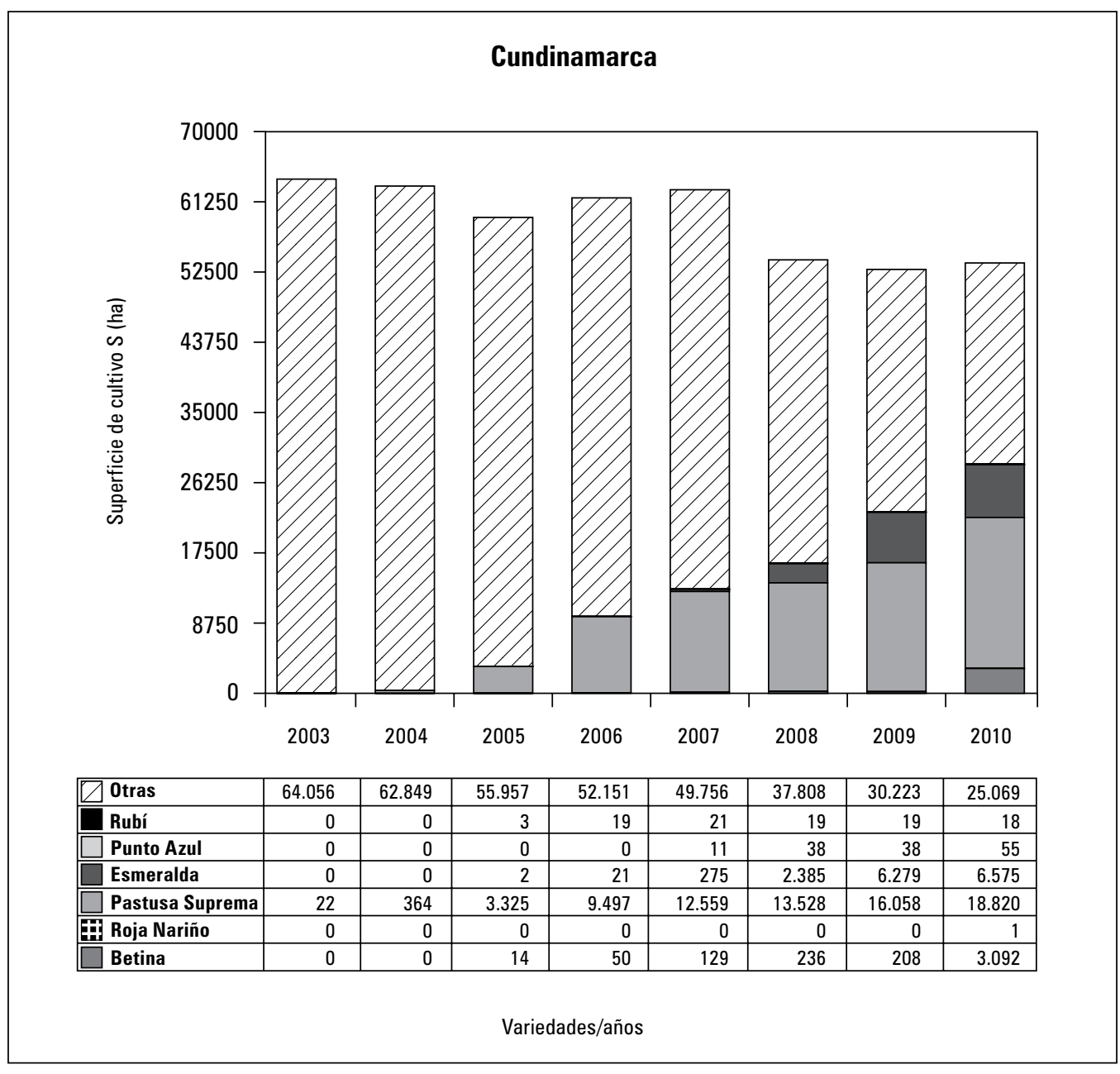

Figura 5. Área de cultivo de nuevas y antiguas (otras) variedades de papa en el departamento Cundinamarca entre 2003 y 2010.

\section{Razones para la adopción y no adopción}

Thurber y Fahey (2009) en el proceso de adopción destacan la importancia de la ventaja relativa de la innovación, su compatibilidad con el adoptador, complejidad de uso, observabilidad de sus resultados, y su capacidad de ser usado experimentalmente. Según Hopkins et al. (2007), la demostración del éxito de una innovación, ir de la teoría a la práctica, tiene un efecto catalizador en la adopción. Para Kshirsagar et al. (2002) la percepción del productor sobre las ventajas y desventajas de la innovación es determinante en la adopción. Brush et al. (1992) ponen de relieve que la adopción está correlacionada con el acceso a la información sobre la innovación, la capacidad de financiar la nueva tecnología, el control del riesgo y su acceso al mercado de factores y productos relacionados con la innovación. Rogers (2003) hace énfasis en los adoptadores, quienes se diferencian por su estatus socioeconómico, valores personales y comportamientos de comunicación. Para Feder et al. (2004), la comunicación y el interés de cooperación tiene gran valor, especialmente para la difusión de innovaciones entre productores. Monge et al. (2008) resaltan la capacidad de persuasión del agente de cambio (promotor de la innovación), la influencia social y la competencia entre adoptadores. En resumen, ventaja clara, practicidad en el uso, acceso e información disponible sobre el nuevo producto son condiciones de adopción. Para la adopción de las nuevas variedades han sido rele- 
vantes sus ventajas, la disponibilidad de semilla e información sobre ellas.

Las características de las nuevas variedades frente a las variedades antiguas son: (1) mayores rendimientos, (2) mayor resistencia a enfermedades, en particular a la "gota", (3) diferentes rangos de adaptabilidad por altitud, (4) apreciable calidad culinaria y (5) buena aptitud para industria. Estas características técnicas con efectos socioeconómicos y medioambientales importantes son consideradas buenas razones para su adopción.
Sin embargo, solo dos han sido relevantes para la adopción por los productores: el incremento de rendimiento (de 25 a $35-40 \mathrm{tha}^{-1}$ ) y la resistencia a enfermedades que determina menor cantidad de pérdidas. De lado del mercado, la denominada demanda creciente ha sido relevante solo para la adopción de Pastusa Suprema.

La no adopción tienen dos razones principales: desconocimiento de las variedades por parte de los productores y falta de disponibilidad de semi1la. La no promoción formal de las nuevas varie-

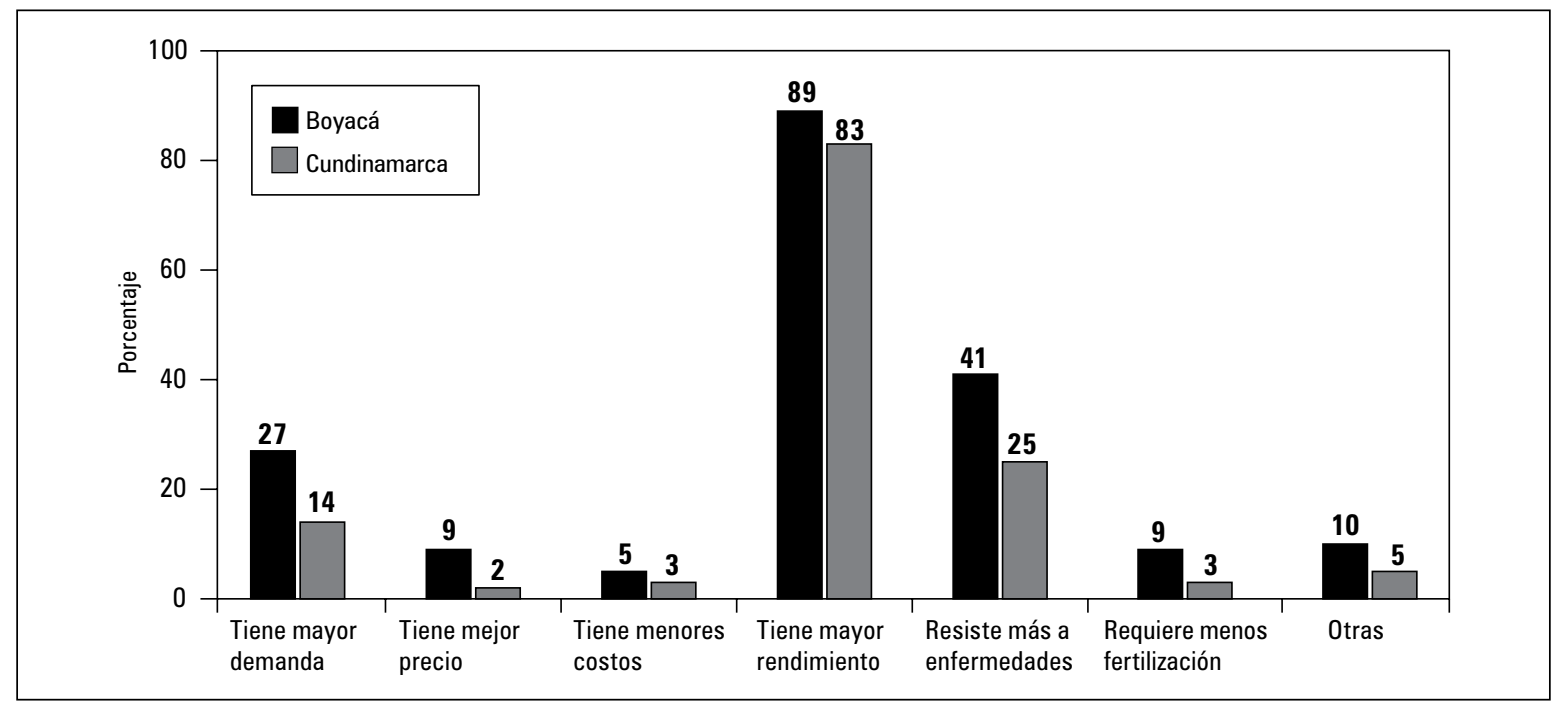

Figura 6. Razones que determinaron la adopción de las nuevas variedades de papa frente a las antiguas en Boyacá y Cundinamarca, entre 2003 y 2010.

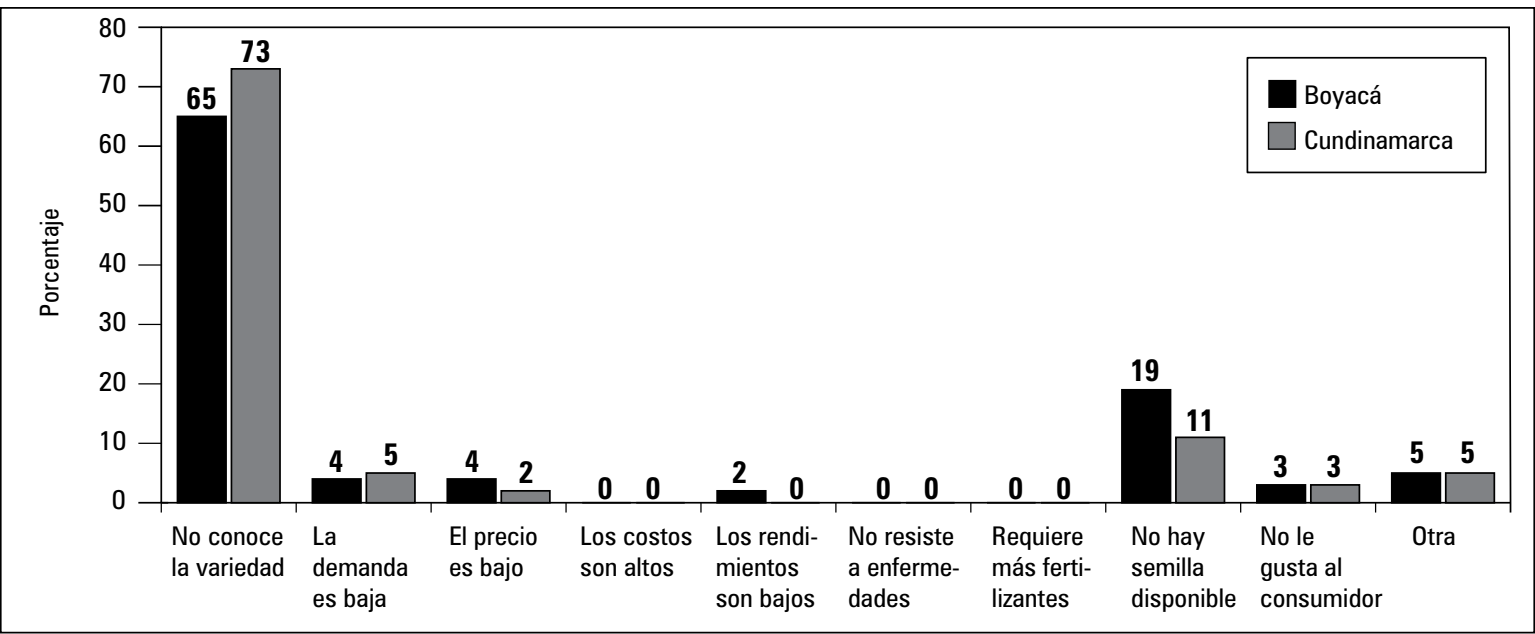

Figura 7. Razones que determinaron la no-adopción de las nuevas variedades de papa frente a las antiguas en Boyacá y Cundinamarca, entre 2003 y 2010. 
dades para productores y consumidores hace que su difusión pase en su mayor parte a manos del mercado, es decir, a la demanda del productor por semilla y del consumidor final por producto (fresco o procesado); a la oferta del producto por parte de los productores y al precio que los comerciantes les ofrezcan.

\section{Proyección de la adopción}

La adopción de las nuevas variedades sigue, de manera general, una función en forma de " $S$ " (Rogers, 2003). Al inicio es lenta, luego se incrementa notablemente, y después disminuye hasta finalizar con cero incrementos. Pastusa Suprema estaría en su tercera fase. Se espera que sus incrementos, $26 \%$ en Boyacá y $17 \%$ en Cundinamarca (periodo 2009-2010) vayan disminuyendo con el tiempo. Esmeralda y Betina estrían ingresando a la segunda fase; mientras que Punto Azul, Rubí y Roja Nariño aún estarían en una primera fase. El incremento de estos últimos grupos dependerá de cómo reacciona el mercado: especialmente la demanda y los precios. Factores técnicos como la resistencia a enfermedades y los buenos rendimientos también serán decisivos en la adopción.

\section{CONCLUSIONES}

- La difusión de las nuevas variedades sigue canales poco convencionales, que obedecen más a directrices de mercado que a políticas públicas. Se han identificado seis canales: (1) productores de papa grandes y medianos, (2) almacén de insumos agropecuarios (Fedepapa), (3) organizaciones de productores de papa, (4) empresa productora de semillas, (5) grupo de investigación en papa y (6) pequeños productores. El canal número uno es el que mayor efecto ha tenido en la difusión.

- Las principales limitantes de la difusión de las nuevas variedades son: la escasa información que les llega a los consumidores y productores sobre las nuevas variedades, baja disponibili- dad de semilla certificada y el poder de negociación de mayoristas y comerciantes intermediarios, quienes fijan precios bajos para las nuevas variedades.

- Pastusa Suprema es la variedad más difundida y adoptada, le siguen Esmeralda y Betina. Con significativamente menores porcentajes de difusión y adopción están Punto Azul, Rubí y Roja Nariño. Esta última casi no se la conoce en los departamentos de estudio.

- La superficie total de cultivo de las nuevas variedades se ha incrementado de forma sostenida hasta alcanzar en 2010 un 43\% de la superficie total de papa en Boyacá y 53\% en Cundinamarca. Pastusa Suprema ocupa el mayor porcentaje de superficie entre el grupo de las nuevas variedades.

- Las nuevas variedades han sido adoptadas principalmente por sus altos rendimientos y por su mayor resistencia a enfermedades respecto de las antiguas. Las razones de su no adopción son la falta de información a nivel de agricultores y la poca semilla disponible.

- Las proyecciones de difusión de las nuevas variedades son diferentes en cada caso. El incremento de Pastusa Suprema irían en descenso; mientras que de Esmeralda y Betina irían en ascenso. Punto Azul, Rubí y Roja Nariño aún están en una fase de lento crecimiento en su difusión.

\section{AGRADECIMIENTOS}

La presente investigación se realizó como una actividad del proyecto "Red de innovación de investigación y desarrollo: hacia la diseminación eficiente y mecanismos de impacto propobre con nuevas variedades de papa en la zona andina", dentro del marco de la Red LatinPapa coordinada desde el Centro Internacional de la Papa (CIP) por el Dr. Stef De Haan, y con el apoyo económico del INIA (España) y el Fondo Fontagro. 


\section{REFERENCIAS BIBLIOGRÁFICAS}

Acasio, R. F., A. De los Santos y P. Van der Zaag. 1986. Acceptance of diffused light storage technology by potato farmers in the benguet and mountain provinces of The Philippines. Amer. Potato J. 63(3), $162-170$

Barrientos, J.C. y E. Berg. 2013. Impact assessment of agricultural innovations: A review. Agron. Colomb. 31(1), 120-130.

Bejarano, C., R. Oros y P. Nava. 2004. Impacto económico del manejo integrado de polilla de la papa. Caso específico: Municipio de Tarabuco. Fundación PROINPA, Cochabamba, Bolivia.

Brush, S.B., J.E. Taylor y M.R. Bellon. 1992. Technology adoption and biological diversity in Andean potato agriculture. J. Develop. Econ. 39 (365-387).

CNP, Consejo Nacional de la Papa. 2010. Información papa. CNP, Bogotá.

Encuesta Nacional Agropecuaria. 2009. Oferta agropecuaria: ENA-Cifras 2009. Ministerio de Agricultura y Desarrollo Rural-Corporación Colombia Internacional, Bogotá.

Esprella, R., J. Blajos, R. Calderon y G. Thiele. 2002. Evaluación de la adopción e impacto económico del manejo integrado del gorgojo en el Altiplano Central. Fundación PROINPA, Cochabamba, Bolivia.

FAO. 2009. Año Internacional de la Papa 2008: Nueva luz sobre un tesoro enterrado. Organización de las Naciones Unidas para la Agricultura y la Alimentación, Roma.

FAOSTAT. 2013. Dirección de Estadística. En: http://faostat.fao.org/DesktopDefault. aspx?PageID=567\&lang=es\#ancor; consulta julio de 2013.

Fedepapa - Secretaría Técnica CNP. 2010. Distribución del área sembrada de papa en Colombia por variedades, 2009. Bogotá.

Feder, G., R. Murgai y J.B. Quizon. 2004. The acquisition and diffusion of knowledge: the case of pest management training in farmer field schools, Indonesia. J. Agr. Econ. 55(2), 221-243.

Hopkins, B.G., D.A. Horneck, M.J. Pavek, B.D. Geary, N.L. Olsen, J.W. Ellsworth, G.D. Newberry, J.S. Miller, R.E. Thornton y G.W. Harding. 2007. Evaluation of potato production best management practices. Amer. J. Potato Res. 84(1), 19-27.
Kshirsagar, K.G., S. Pandey y M.R. Bellon. 2002. Farmer perceptions, varietal characteristics and technology adoption: a rainfed rice village in Orissa. Econ. Pol. Weekly 37(13), 1239-1246.

Mauceri, M., J. Alwang, G. Norton y V. Barrera. 2005. Adoption of integrated pest management technologies: A case study of potato farmers in Carchi, Ecuador. Memorias Annual Meeting American Agricultural Economics Association, Providence (RI), July 24-27, 2005.

Observatorio Agrocadenas Colombia. 2005. La cadena de la papa en Colombia: Una mirada global de su estructura y dinámica 1991-2005. Documento de Trabajo No. 54. Ministerio de Agricultura y Desarrollo Rural, Bogotá.

Ministerio de Agricultura y Desarrollo Rural, DNP y DANE. 2003a. I Censo nacional de la papa Departamento de Cundinamarca. Consolidado de la muestra de producción y rendimiento - áreas cosechadas. Año agrícola julio del 2001 a junio del 2002. Bogotá.

Ministerio de Agricultura y Desarrollo Rural, DNP y DANE. 2003b. I Censo nacional de la papa Departamento de Boyacá - Separata V. Consolidado de la muestra de producción y rendimiento - áreas cosechadas. Año agrícola junio del 2002 a junio del 2003. Bogotá.

Monge, M., F. Hartwich y D. Halgin. 2008. How change agents and social capital influence the adoption of innovations among small farmers: evidence from social networks in rural Bolivia. International Food Policy Research Institute, Discussion Paper 00761, Washington DC.

Morris, M., M. Mekuria y R. Gerpacio. 2003. Impacts of CIMMYT maize breeding research. pp. 135-159 En: Evenson, R.E. y D. Gollin (eds.). Crop variety improvement and its effect on productivity: The Impact of International Agricultural Research. FAO, London.

Nústez, C.E. 2011.Variedades colombianas de papa. Facultad de Agronomía, Universidad Nacional de Colombia, Bogotá.

Rogers, E.M. 2003. Diffusion of innovations. 5th ed. Free Press, New York, NY.

Thurber, M.D. y J.W. Fahey. 2009. Adoption of Moringa oleifera to combat under-nutrition viewed through the lens of the "Diffusion of Innovations" theory. Ecol. Food Nutr. 48(3), 212-225. 